\title{
Tailoring the emission spectrum of colloidal nanocrystals by means of lithographically-imprinted hybrid vertical microcavities
}

\author{
Luigi Martiradonna ${ }^{* a, b}$, Luigi Carbone ${ }^{\text {a, }}$, Milena De Giorgi ${ }^{a}$, Tiziana Stomeo ${ }^{\text {a, }}$, \\ Maria Teresa Todaro ${ }^{\mathrm{a}}$, Marco Anni ${ }^{\mathrm{a}}$, Liberato Manna ${ }^{\mathrm{a}}$, Giuseppe Gigli ${ }^{\mathrm{a}}$, \\ Roberto Cingolani ${ }^{\mathrm{a}}$ and Massimo De Vittorio ${ }^{\mathrm{a}}$ \\ ${ }^{a}$ National Nanotechnology Laboratory of INFM (NNL-INFM), Distretto Tecnologico-ISUFI, \\ Università di Lecce, Via Arnesano, 73100 Lecce, ITALY \\ ${ }^{\mathrm{b}}$ ISUFI, Istituto Superiore Universitario per la Formazione Interdisciplinare, Via Arnesano, 73100 \\ Lecce, ITALY
}

\begin{abstract}
Colloidally synthesized CdSe/ZnS core/shell semiconductor nanocrystals (NCs) show highly efficient, narrow-width and size-tunable luminescence. Moreover, they can be incorporated in polymer matrices and deposited on solid substrates by means of spin-coating techniques. When embedded between two mirrors a NCs/polymer blends microcavity is realised, thus allowing to tailor the photoluminescence spectrum of these emitters. By virtue of the quantized photonic and electronic density of states, colloidal quantum dots embedded in a single mode vertical microcavity are good candidates for the fabrication of high-efficiency emitting devices with high spectral purity and directionality.

In this paper, we have applied a new organic-inorganic hybrid technology for the fabrication by imprint lithography (IL) of vertical microcavities that embed colloidal quantum dots.

Two dielectric distributed Bragg reflectors (DBR) are evaporated on two different substrates. The active organic layer (NCs/polymer blend) is spin coated on the first DBR, whereas a lithographic pattern is realized on the second DBR, used as the IL mold. The two parts are then assembled together in an IL process in order to create a vertical microcavity. The fine control of the thickness of the active material waveguide layer can be achieved through the mold patterning depth and the IL process parameters. All the fabrication steps have been engineered in order to decrease the thermal stress of the active layer.

The effectiveness of this technology is demonstrated by the room temperature photoluminescence (PL) spectra, recorded on the fabricated microcavity, which show a sharp emission peak with a line width of $4.15 \mathrm{~nm}$.
\end{abstract}

Keywords: Colloidal nanocrystals, CdSe/ZnS, thin films, quantum dots, imprint lithography, hybrid microcavity

\section{INTRODUCTION}

Colloidal nanocrystals (NCs) are an important class of materials in nanoscience and nanotechnology. They are chemically synthesized in solution at low cost and in large numbers ${ }^{1,2}$, with a full control on size and shape. By virtue of the three-dimensional electronic confinement, the density of states is quantized and the emission spectrum of an ensemble of NCs shows a typical sharp peak with a full width at half maximum (FWHM) of few tens of nanometers. Moreover, the central emission wavelength can be finely tuned in the whole visible spectral range by controlling the NCs size. This high versatility has fostered the application of nanocrystals in diverse technological fields such as lightemitting diodes (LEDs) ${ }^{3}$, sensing ${ }^{4-6}$, photovoltaics ${ }^{7,8}$ and electronics 9 .

Several proposed applications of nanocrystals are in the area of optical devices. In fact NCs can be incorporated in polymer matrices and deposited on solid substrates by means of spin-coating techniques. Vertical microcavities embedding thin films of NCs between two mirrors can be realized, thus allowing to tailor the photoluminescence spectrum of these emitters ${ }^{10-12}$. The impact of planar vertical microcavities on the spontaneous emission of active layers

luigi.martiradonna@unile.it; phone:+39.0832.298383; fax:+39.0832.298237; www.nnl.it 
has been widely studied ${ }^{13}$, leading to the achievement of sharp directionality, spectral narrowing and photoluminescence enhancement at the resonant wavelengths. Tunable LEDs with high directionality and high spectral purity can be fabricated. Moreover, since stimulated emission and optical gain in colloidal NCs have been proved ${ }^{14}$, highly performing hybrid lasers could be obtained in principle. Microcavities are also widely used in the investigation of light-matter interaction phenomena, such as Rabi splitting in strong coupling regime ${ }^{15}$ and enhancement of Förster energy transfer between semiconducting nanocrystals and active polymers ${ }^{16,17}$.

In this paper we report a new technique for the fabrication of hybrid organic-inorganic vertical microcavities which embed a thin film of polymer-nanocrystals blend between two dielectric Distributed Bragg Reflectors (DBRs). Various works have reported similar hybrid devices ${ }^{10-12,18}$, but they all show a weak control over the distance between the two mirrors. This distance, however, is a fundamental parameter of microcavities as it determines the resonance wavelength of the structure. In our devices, this control is achieved by means of an unconventional use of the imprint lithography (IL) technique. Moreover, in our approach the active blend does not undergo very high process temperatures, and therefore it avoids any degeneration of the emission properties from the blend.

Poly(methylmethacrylate) (PMMA) has been used as the transparent polymer in which CdSe nanocrystals with a $\mathrm{ZnS}$ shell have been dispersed. The two DBRs have been separately prepared by evaporating (with electron-beam lithography) alternated quarter-wave thick layers of $\mathrm{TiO}_{2}$ and $\mathrm{SiO}_{2}$. The active organic layer has been spin coated on the first DBR, whereas a $\lambda$-thick SU8 lithographic pattern has been realized on the second DBR. The latter DBR is used as the IL mold but also as a spacer between the two mirrors. The two parts have then been assembled together in an IL process in order to create the vertical microcavity. The fine control of the thickness of the active material waveguide layer is achieved through the mold patterning depth.

Room-temperature photoluminescence (PL) spectra have been recorded in order to verify the tailoring effects of the resonant cavity on the nanocrystals. A decrease of the NCs emission FWHM from $40 \mathrm{~nm}$ to only $4 \mathrm{~nm}$ has been achieved with our devices.

\section{FABRICATION OF THE DEVICE}

\subsection{Processing guidelines}

The realization of hybrid organic-inorganic vertical microcavities based on imprint lithography tools has been previously demonstrated with organic emitters ${ }^{19}$ and it showed its effectiveness, as it allowed to fabricate vertical cavities with a Qfactor equal to 112. This work reports the extension of this approach, for the first time, to inorganic emitters (CdSe/ $\mathrm{ZnS}$ core/shell nanocrystals) dispersed in a polymer matrix.

Two DBR reflectors were prepared by e-beam evaporation of $\mathrm{SiO}_{2}$ and $\mathrm{TiO}_{2}$ on silicon and quartz substrates. As opposed to metallic mirrors, distributed Bragg reflectors are not absorbing and they can be designed in order to reflect a limited range of wavelengths (the DBR stop-band) while being transparent for wavelengths not belonging to the stop-band. Therefore, DBRs can act as cavity mirrors for the NCs emission wavelengths, while allowing the optical excitation of the dots via an external ultraviolet source. Moreover, metallic mirrors have typical reflectivities of $90 \%$, whereas DBRs can reach values close to unity. High reflectivities are essential for the realization of high-performing microcavities. It is well known in fact that the line width (or the full width at half maximum, FWHM) of the resonance dip decreases as the reflectivity of the mirrors increases, with a resulting enhancement of the spectral purity. The FWHM is expressed by:

$$
\Delta \lambda_{\text {FWHM }}=\frac{\lambda^{2}}{2 n L_{c} \pi}\left(1-\sqrt{R_{1} R_{2}}\right) .
$$

$n$ is the refractive index of the active material, $L_{C}$ is the cavity length (that is the distance between the mirrors), $\lambda$ is the emission wavelength, and $R_{l}$ and $R_{2}$ are the power reflectivities of the mirrors.

A thin film of the polymer-NCs blend has been spin coated on the first DBR, with a thickness approximately equal to the cavity length. The mechanical properties of the active material have been investigated in order to verify the suitability of the matrix for the spin-coating and for the imprint lithography processes. More specifically, an important property is the matrix viscosity, which determines the thickness of the spin-coated layer and the flow of the soft material into the mold recesses during the imprint process. Therefore the choice of NCs and polymer concentrations in the solvent has thus been made in order to obtain a viscosity suitable for the spin-coating as well as for the imprint steps. Another mechanical critical parameter is the glass transition temperature $\left(\mathrm{T}_{\mathrm{G}}\right)$ of blend, which determines the imprint assembly process temperature ${ }^{20}$. In our devices we have used poly(methylmethacrylate) (PMMA) diluted in chlorobenzene $\left(\mathrm{C}_{6} \mathrm{H}_{5} \mathrm{Cl}\right)$ as the polymer host for nanocrystals, since its mechanical behaviour is well known ${ }^{19,20}$. 
A layer of SU8-2000 negative resist has been spin coated on the second DBR and it has lithographically patterned and cured in order to create a mold for the subsequent imprint process. The pattern consisted of a 1D periodic sequence of 25 $\mu \mathrm{m}$ wide stripes, with a period of $250 \mu \mathrm{m}$. The mold became part of the final sandwiched structure, acting as a spacer between the two DBRs. Its depth was therefore responsible for the microcavity length $\mathrm{L}_{\mathrm{C}}$. The glass-transition temperature $\mathrm{T}_{\mathrm{G}}$ of cured Su8 is higher than $200^{\circ} \mathrm{C}$, so that the mold could bear the typical temperatures of IL processes $\left(<200^{\circ} \mathrm{C}\right)$ without undergoing permanent deformations.

The imprint process has been used to assemble the vertical microcavity, formed by the active layer sandwiched between the two high reflectivity DBRs. Figure 1a shows a scheme of the two DBRs before the realization of the vertical microcavity. In figure $1 \mathrm{~b}$ the complete sandwiched structure is shown, obtained after pressing the two parts with imprint lithography tools.

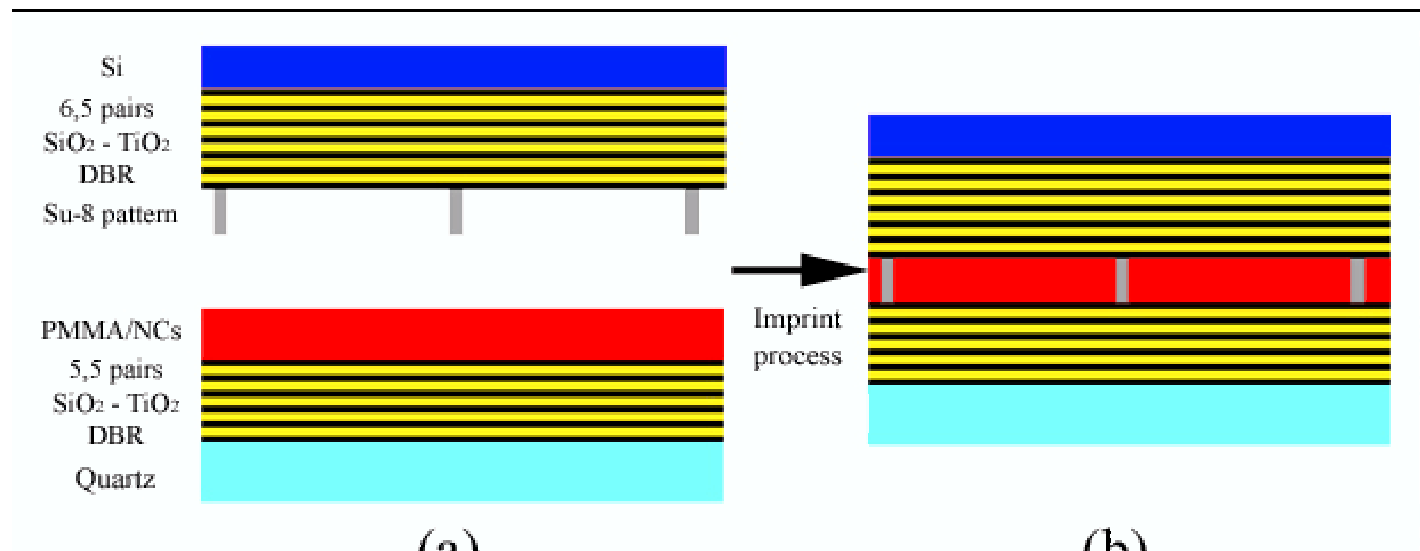

(a)

(b)

Fig.1: (a) Scheme of the two dielectric DBR covered by a patterned and cured SU8-2000 resist and by a layer formed by PMMA and nanocrystals, respectively. (b) The complete sandwiched vertical cavity structure obtained by imprint lithography.

The main innovation of this technique is the possibility to control the cavity length by simply varying the thickness of the SU8 pattern. Moreover, the mold pattern generates refractive index discontinuities along the active material plane, which create a photon confinement also in these directions. A straightforward development of this technique is an effective optical lateral confinement of the active material: the pattern of the vertical spacer can be designed in order to realize a two-dimensional photonic crystal structure that reflects the in-plane NCs emission wavelength. Three-dimensional microcavities with very sharp resonance dips could be in principle realized by this method. A more detailed description of the different parts is given in the following subsections.

\subsection{Fabrication of the NCs/polymer blend and of the DBR mirrors}

Nearly spherical, core/shell CdSe/ZnS nanocrystals were prepared using standard methods ${ }^{21,22}$. The nanocrystals are coated with a layer of surfactants, namely trioctylphosphine oxide (TOPO), and trioctylphosphine (TOP), which determine their solubility in non-polar or moderately polar solvents.

Figure 2 reports the emission (solid line) and absorption (dashed line) spectra of CdSe/ZnS core/shell nanocrystals used in our devices. The emission peak is centered at $580 \mathrm{~nm}$ and has a FWHM of $40 \mathrm{~nm}$. The typical absorption spectrum of nanocrystals shows that these emitters can be optically pumped efficiently in the ultra-violet spectral range, far from their emission region. 


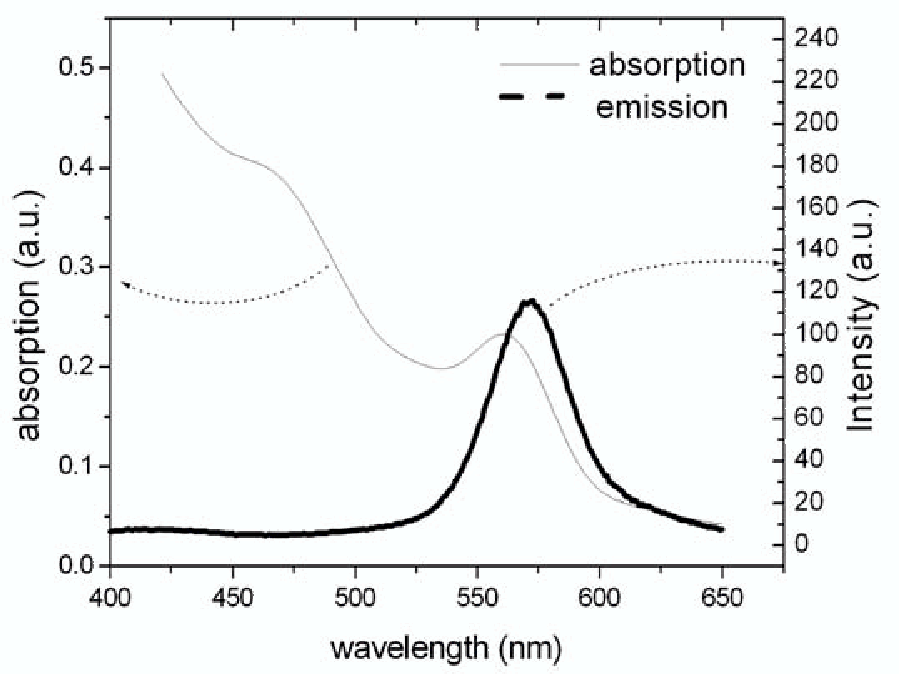

Fig.2: Emission and absorption spectra of the $\mathrm{CdSe} / \mathrm{ZnS}$ core/shell colloidal nanocrystals used in this work.

NCs have been dispersed in a matrix of poly(methylmethacrylate) (with molecular weight $\mathrm{M}_{\mathrm{r}}=950.000$ ) diluted in chlorobenzene $\left(\mathrm{C}_{6} \mathrm{H}_{5} \mathrm{Cl}\right)$. This polymer is transparent to ultra-violet and visible radiations, and therefore it does not influence the optical properties of the NCs. The choice of PMMA is due to its well known thermo-plastic properties ${ }^{19,20}$ which make this polymer well suited for spin-coating and imprint lithography processes. In particular, typical layers with thicknesses ranging from $50 \mathrm{~nm}$ to $500 \mathrm{~nm}$ can be obtained by using a weight concentration of $\mathrm{PMMA}$ in $\mathrm{C}_{6} \mathrm{H}_{5} \mathrm{Cl}$ ranging from $2 \%$ to $4 \%$. The glass transition temperature of PMMA-950K is about $140^{\circ} \mathrm{C}$.

The concentration of polymer and NCs in chlorobenzene has been chosen in order to optimize three critical aspects, namely the viscosity of the solution, the layer roughness and the photoluminescence efficiency.

The photoluminescence efficiency $\left(\eta_{\mathrm{PL}}\right)$ of NCs is basically conditioned by the NCs-NCs interaction and by the NCsPMMA interaction. A high concentration of PMMA can lead to a reduction of photoluminescence efficiency, because methacrylate groups can interact with the surfactants surrounding the nanocrystals, thus modifying their optical properties. At the same time, dense solutions of nanocrystals can lead to concentration quenching, due to a non-radiative energy transfer among the clusters and a consequent decrease of the efficiency.

\begin{tabular}{|c|c|c|c|}
\hline Sample & $\eta_{\mathrm{PL}}(\%)$ & Error on $\eta_{\mathrm{PL}}(\%)$ & Surf. roughness $(\mathrm{nm})$ \\
\hline 1. NCs/PMMA X $=822.5$ & 1.032 & \pm 1.293 & 5.23 \\
\hline 2. NCs/PMMA X $=1645$ & 2.978 & \pm 1.766 & - \\
\hline 3. NCs/PMMA X $=2470$ & 0.9403 & \pm 1.146 & - \\
\hline 4. NCs/PMMA X $=3290$ & 2.1792 & \pm 0.338 & 13.5 \\
\hline 5. NCs/PMMA X $=6580$ & 1.9067 & \pm 0.208 & - \\
\hline 6. NCs/PMMA X $=9870$ & 1.6077 & \pm 0.167 & 39.7 \\
\hline
\end{tabular}

Table 1: Photoluminescence efficiency $\left(\eta_{\mathrm{PL}}(\%)\right)$ and surface roughness for a series of NCs/PMMA thin films. In the preparation of these films, the molarity of PMMA is kept constant $\left(\mathrm{M}_{\mathrm{PMMA}}=3.492^{*} 10^{-5} \mathrm{~mol} / \mathrm{l}\right)$, while the NCs molarity is varied from $\mathrm{M}_{\mathrm{NCs}}=$ $2.8709^{*} 10^{-2} \mathrm{~mol} / 1$ (in sample 1 ) to $\mathrm{M}_{\mathrm{NCs}}=3.4451 * 10^{-1} \mathrm{~mol} / \mathrm{l}$ (in sample 6).

Photoluminescence efficiency measurements on NCs/PMMA thin films spin-coated on quartz substrates have been carried out according to the method proposed by Greenham et al. ${ }^{23}$. The concentration of PMMA in chlorobenzene was kept constant $\left(\mathrm{M}_{\mathrm{PMMA}}=3.492 * 10^{-5} \mathrm{~mol} / \mathrm{l}\right)$, whereas the molar concentration of the NCs was varied from $\mathrm{M}_{\mathrm{NCs}}=$ $2.8709 * 10^{-2} \mathrm{~mol} / \mathrm{l}$ to $\mathrm{M}_{\mathrm{NCs}}=3.4451 * 10^{-2} \mathrm{~mol} / \mathrm{l}$, i.e. Therefore the molar ratio between NCs and PMMA $\mathrm{X}=M_{N C S} / M_{P M M A}$ was varied from 822,5 to 9870 . The values of the photoluminescence efficiency $\left(\eta_{\mathrm{PL}}(\%)\right)$ for the various concentrations are reported in Table 1 . Due to the low concentration of the emitters in the host, the signal 
detected from samples with a molar ratio of $822.5,1645$ and 2470 was comparable with the environmental noise. When the molar ratio was increased from 3290 to 9870 , the photoluminescence efficiency slightly decreased, probably due to concentration quenching effect. The highest, reproducible, value for $\eta_{\mathrm{PL}}$ was therefore equal to $2.18 \%$ which was recorded from the sample with a molar ratio equal to 3290 .

Atomic Force Microscopy (AFM) studies have been carried out on samples prepared at $\mathrm{X}=822.5, \mathrm{X}=3290$ and $\mathrm{X}=$ 9870, in order to evaluate the surface roughness of the deposited films (fig.3).

Figure 3a refers to the NCs/PMMA blend with $X=822.5$; figure $3 b$ refers to the $X=3290$ sample; figure $3 \mathrm{c}$ refers to the $X=9870$ sample. The scanned area was $5 \mu \mathrm{m} \times 5 \mu \mathrm{m}$. In these figures clusters of increasing size can be seen. These clusters are probably formed from the interaction between the polymer chains and the surfactants that coat the nanocrystals surface, so that PMMA chains aggregate in big and irregular groups. Root mean square roughnesses, measured over areas $1 \mu \mathrm{m} \times 1 \mu \mathrm{m}$ wide, correspond to $5.23 \mathrm{~nm}, 13.5 \mathrm{~nm}$ and $39.7 \mathrm{~nm}$ for figure $3 \mathrm{a}, 3 \mathrm{~b}$ and $3 \mathrm{c}$ respectively. As expected, the surface roughness increases as the concentration of NCs in the blend is increased.

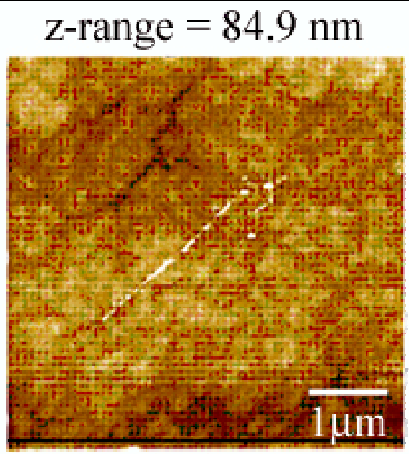

(a)

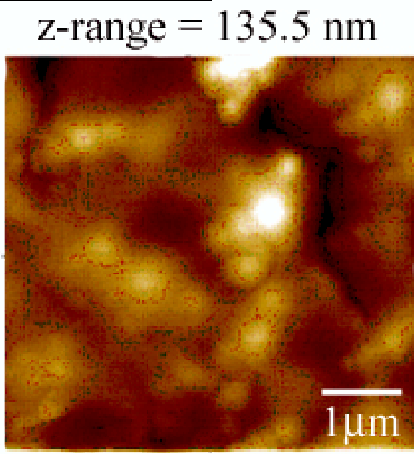

(b)

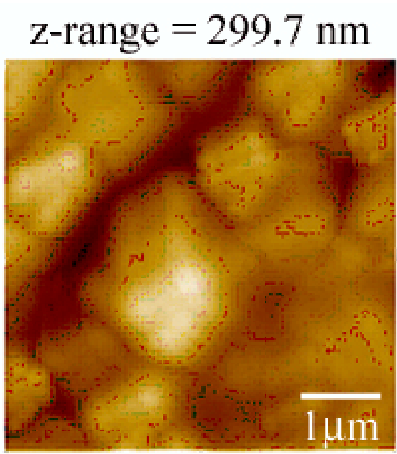

(c)

Fig.3: AFM images of thin films, each $300 \mathrm{~nm}$ thick, spin-coated on quartz substrates. The deposited solutions are MCs/PMMA blends in Chlorobenzene with a PMMA concentration of $\mathrm{M}_{\mathrm{PMMA}}=3.492 * 10^{-5} \mathrm{~mol} / \mathrm{l}$ and a NCs concentration of $\mathrm{M}_{\mathrm{NCs}}=2.8709 * 10^{-2}$ $\mathrm{mol} / \mathrm{l}(\mathrm{a}) \mathrm{M}_{\mathrm{NCs}}=1.1483 * 10^{-1} \mathrm{~mol} / 1$ (b) and $\mathrm{M}_{\mathrm{NCs}}=3.4451 * 10^{-1} \mathrm{~mol} / \mathrm{l}$ (c). Scanned area: $5 \mu \mathrm{m} \times 5 \mu \mathrm{m}$

From these two analyses on the active material concentration, we have chosen a value of $X=3290$, which is a good compromise between the surface roughness and the photoluminescence efficiency.

In order to decrease the imprint process duration, soft materials with low viscosity are preferred ${ }^{20}$. We have tried to reduce the viscosity of our solution by decreasing the weight concentration of the polymer down to $2 \%$ $\left(\mathrm{M}_{\mathrm{PMMA}}=2.328^{*} 10^{-5} \mathrm{~mol} / \mathrm{l}\right)$. Unfortunately, this low concentration did not allow us to spin-coat thin films with a thickness of about $300 \mathrm{~nm}$, which is the expected distance between the DBRs. For this reason, we have decided to use a weight concentration of 3\% of PMMA-950K in chlorobenzene.

Two distributed Bragg reflectors have been deposited by means of electron-beam evaporation of alternated layers of $\mathrm{TiO}_{2}$ and $\mathrm{SiO}_{2}$. The evaporation has been carried out at $240^{\circ} \mathrm{C}$ to improve the uniformity of the layers. For the back mirror a 6,5 pairs of $\mathrm{TiO}_{2}-\mathrm{SiO}_{2}$ deposited on a $\mathrm{Si}$ substrate has been chosen, with a reflectivity of $98 \%$. For the front mirror a 5,5 pairs DBR on a quartz substrate has been chosen, with a reflectivity of $98.32 \%$. The front mirror has a transmission coefficient of $92.42 \%$ at $\lambda=325 \mathrm{~nm}$ and $78.45 \%$ at $\lambda=405 \mathrm{~nm}$, the excitation wavelengths chosen for the following photoluminescence (PL) measurements.

\subsection{Microcavity assembly via imprint process}

The SU8 pattern used as a mold for the imprint process has been realized by means of a photolithography process on the 6,5 pairs DBR. Its thickness has been determined according to the cavity length $\mathrm{L}_{\mathrm{C}}$, which is equal to:

$$
L_{C}=\frac{\lambda_{e m}}{n_{a}} \cong 370 \mathrm{~nm} \text {. }
$$

$\lambda_{e m}$ is the active material emission peak and $n_{a}$ is the refractive index of the active material. 
Two different molds have been used, one $370 \mathrm{~nm}$ thick and the other $330 \mathrm{~nm}$ thick, respectively. The first thickness has been chosen as to match exactly $\mathrm{L}_{\mathrm{C}}$, under the assumption of a complete penetration of the pattern into the active layer. The second thickness, on the other hand, has been chosen under the assumption that the soft material is not completely removed during the imprint process, so that a bottom layer remains under the mold ${ }^{20}$. In the latter case, the distance between the two mirrors is determined as the sum of the mold thickness plus the thickness of the bottom layer.

On the second DBR, the active layer has been spin-coated without any baking process, in order to reduce the thermal stresses. The initial thickness of the blend was lower than the requested $370 \mathrm{~nm}$ because a redistribution of the soft material from the regions under the mold toward the recessed regions was taken into account.

The 5,5 pairs DBR was $2 \mathrm{~cm} \times 2 \mathrm{~cm}$ wide, whereas the 6,5 pairs DBR was cleaved to an area $3 \mathrm{~mm} \times 3 \mathrm{~mm}$ wide, in order to improve the uniformity of the pressure during the imprint process.

The imprint press used for the assembly of the cavity has been heated at $180^{\circ} \mathrm{C}$, which is a process temperature higher than the glass transition temperature of PMMA $\left(140^{\circ} \mathrm{C}\right)$ but lower than that of the SU8 mold $\left(200^{\circ} \mathrm{C}\right)$. After this treatment, the two parts have been loaded in the press and a force has been applied for 5 minutes. The system has been cooled down below the $\mathrm{T}_{\mathrm{G}}$ of PMMA without removing the mold.

Three different samples microcavities have been realized, with different mold thicknesses and applied forces: sample (A) with mold thickness of $370 \mathrm{~nm}$ and force of $2 \mathrm{KN}$; sample (B) with mold thickness of $330 \mathrm{~nm}$ and a force of $2 \mathrm{KN}$ and sample (C) with mold thickness of $330 \mathrm{~nm}$ and a force of $3 \mathrm{KN}$.

Room-temperature photoluminescence measurements have been performed in order to test the performances of the three microcavities. A He-Cd laser source operating at $325 \mathrm{~nm}$ with $35 \mathrm{~mW}$ output power was focused on a $100 \mu \mathrm{m}$ wide spot on the sample with a NA $=0.18$ quartz lens. The sample emission from the microcavity was collected in backscattering configuration through the same lens and dispersed by a $30 \mathrm{~cm}$ monochromator equipped with a CCD sensor. The sample was mounted on a microtranslation stage in order both to map the surface emission and to verify the device homogeneity. The results obtained from the PL analysis have been reported in the following section.

\section{RESULTS AND DISCUSSION}

We have detected various photoluminescence emission peaks when positioning the excitation beam in equally spaced points of the microcavities. In each of the three samples we have found that the peak position shifts periodically from $560 \mathrm{~nm}$ to $640 \mathrm{~nm}$ both in the directions perpendicular and parallel to the mold stripes. These results have allowed us to infer the vertical profile of the different samples and the uniformity of the imprint assembly.

A typical vertical profile of the three samples in the direction perpendicular to the stripes is shown in fig.4.

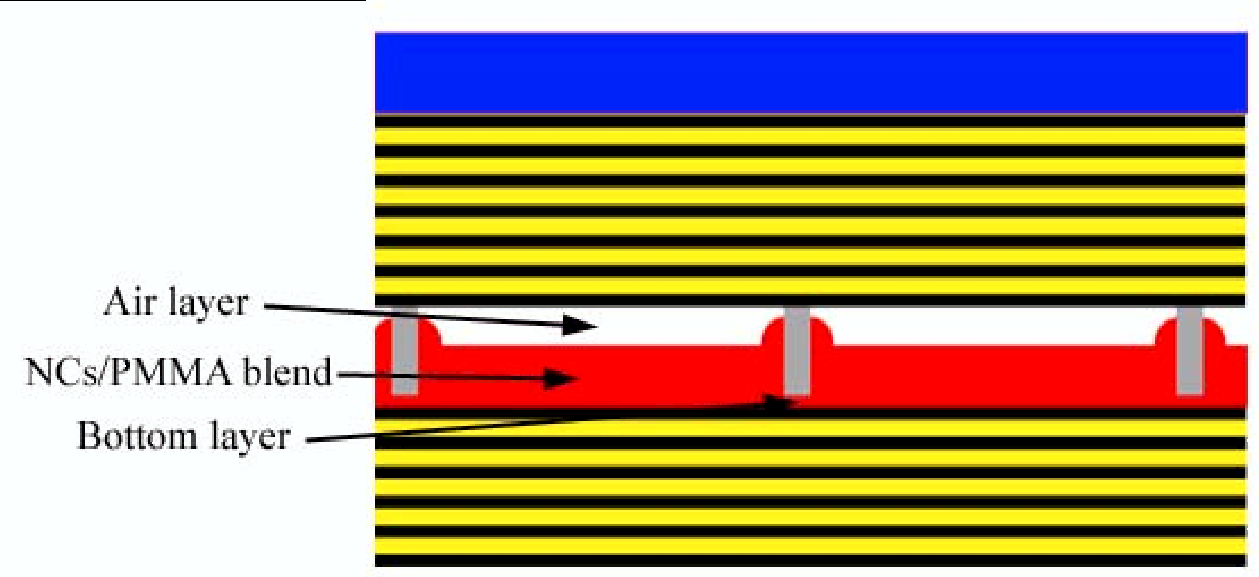

Fig. 4: Section of the microcavities showing the different NCs/polymer blend heights along the structure. The presence of a bottom layer and an air layer trapped in the device are also visible.

According to the filling mechanism described by Heyderman et al.$^{20}$, the SU8 pattern penetrates into the soft material, which fills the recessed regions of the mold. The NCs/polymer blend moves laterally into the cavity and creates raised regions at the borders. The central cavity region is slightly interested by this flow, so that a thin air layer is trapped 
between the active layer and the upper DBR. The mold penetration is not complete but a residual bottom layer of soft material is left under the Su8 pattern. Even if the physical distance between the mirrors remains the same along this section, the resonance wavelength varies due to the different refractive indices of the active material, the Su8 stripes and the air layer. We can thus distinguish three main regions having three different emission peaks positions: the region under the mold, the border cavity region near the mold and the central cavity region.

We have attributed the spectral shift of the peaks along the direction parallel to the Su8 stripes to the non perfect parallelism between the two DBRs forming the microcavity. In fact, the thicknesses of the bottom layer, the raised soft material and the trapped air depend on the imprint process. Slightly non parallel plates thus lead to a thickness gradient and to a shift of the resonance wavelength along the surface. We have observed this effect on sample (A). By moving the excitation source on different regions of the sample, the emission peaks show a spectral shift of about $25 \mathrm{~nm}$ per mm. This would correspond to a thickness gradient of $40 \mathrm{~nm}$ per $\mathrm{mm}$. The two assembled DBRs are therefore affected by a misalignment of $0.0023^{\circ}$.

Samples (B) and (C) show both a higher thickness gradient (about 60nm per $\mathrm{mm}$ ). Moreover, each spectrum detected on these samples shows two emission peaks, corresponding to two different resonant cavity modes, both included in the NCs emission spectrum. This is due to a higher distance between the two mirrors, so that higher order oscillating modes with a reduced spectral distance are included in the NCs emission spectrum. The distance between the two resonant emission peaks is of about $60 \mathrm{~nm}$ in sample (B), while it increases to $80 \mathrm{~nm}$ in sample (C). This means that the cavity length has been reduced, thanks to the increased strength applied to sample (C). However, both samples were not monomodal, and this result demonstrates that the control of the cavity length is less efficient if the patterns have thicknesses shorter than $\mathrm{L}_{\mathrm{C}}$.

According to equation 1, the resonance dip line width of the microcavity (which tailors the NCs emission) depends on the reflectivity coefficients or the two mirrors. As a consequence, the emission peaks FWHM varies depending on the central emission wavelength (the DBRs reflectivity is not constant). The sharpest peaks are located in the central region of the DBRs stop bands, where the reflectivity is the highest possible. We have detected a sharp emission peak centered at $606 \mathrm{~nm}$, with a FWHM of $4.15 \mathrm{~nm}$ As it is shown in figure 5, the peak is perfectly centered in the stop bands of the back and front mirrors.

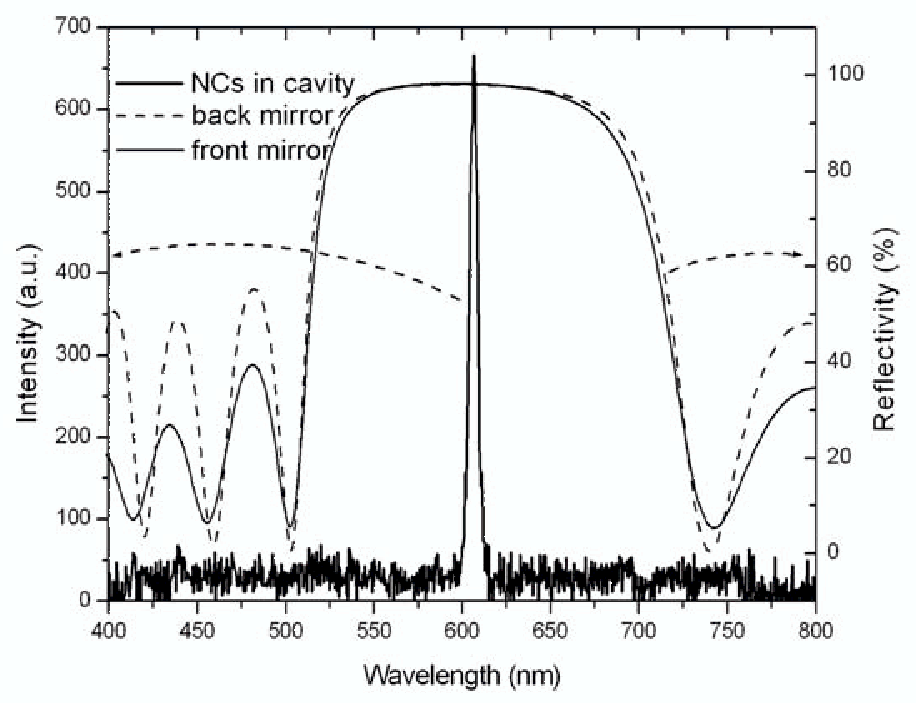

Fig. 5: Comparison between the emission of NCs embedded in the microcavity (bold line) and the reflectivity spectra of the back (dashed line) and front (solid line) mirrors.

The tailoring effect of the microcavity on the NCs spontaneous emission is demonstrated: the initial line width of $40 \mathrm{~nm}$ (see figure 2) is reduced by a factor of 10 .

The measurement of the emission line width is affected by two limitations which lead to an underestimation of the real value. The first limitation is due to the $10^{\circ}$ acceptance cone (half-angle) of the detection system. This should be reduced 
further if we want to avoid spreading of the central peak caused by the dependence of the resonance wavelength from the detection angle. Tessler et al. ${ }^{24}$ reported a reduction of the FWHM measure of the cavity mode from $4 \mathrm{~nm}$ to $1 \mathrm{~nm}$ when using collecting systems with acceptance cones of $8.25^{\circ}$ and $0.4^{\circ}$ (half angle), respectively. The second limitation is related to the variation of the cavity thickness over the excited area, whose diameter was approximately $100 \mu \mathrm{m}$. The thickness gradient of our microcavities causes a spectral shift of the peak of about $25 \mathrm{~nm}$ per mm, thus the detected signal should be a convolution of narrow peaks ${ }^{25}$ with an average shift of $2.5 \mathrm{~nm}$.

\section{CONCLUSIONS AND FURTHER WORK}

We have fabricated hybrid organic-inorganic vertical microcavities by using imprint technology. This technique has been applied for the first time to inorganic emitters (CdSe/ZnS core/shell nanocrystals) dispersed in a polymer matrix. As opposed to commonly fabricated organic vertical cavity devices, this method allows one to carefully control the active material waveguide layer through the control of the DBR/mold patterning depth and through the imprint process.

The effectiveness of this technology has been demonstrated by the room temperature photoluminescence (PL) spectra, recorded on the fabricated microcavity, which show a sharp emission peak with a FWHM of $4.15 \mathrm{~nm}$.

A straightforward development of this technique is the optical lateral confinement of the active material: the pattern of the vertical spacer can be designed in order to realize a two-dimensional photonic crystal reflecting the NCs emission wavelength. Three-dimensional microcavities with even sharper emission peaks could be in principle realized in this way. The study of the optical and electrical properties of nanoparticles confined in nanocavities will be of great interest for the fabrication of extremely low-threshold hybrid lasers. Moreover, these devices promise to be efficient instruments for the investigation of the effects of 3D optical confinement on energy-matter interaction phenomena.

\section{ACKNOWLEDGEMENTS}

The authors gratefully thank Marco Mazzeo and Luca Troisi for fruitful discussions. We also acknowledge the expert technical help of Paolo Cazzato, Gianmichele Epifani and Stefania D'Amone.

\section{REFERENCES}

(1) Kasuya, A.; Sivamohan, R.; Barnakov, Y. A.; Dmitruk, I. M.; Nirasawa, T.; Romanyuk, V. R.; Kumar, V.; Mamykin, S. V.; Tohji, K.; Jeyadevan, B.; Shinoda, K.; Kudo, T.; Terasaki, O.; Liu, Z.; Belosludov, R. V.; Sundararajan, V.; Kawazoe, Y., "Ultra-stable nanoparticles of CdSe revealed from mass spectrometry" Nature Materials, 3, 99-102, 2004

(2) Alivisatos, A. P. "Semiconductor Clusters, Nanocrystals and Quantum Dots" Science, 271, 933-937, 1996.

(3) Tessler, N.; Medvedev, V.; Kazes, M.; Kan, S. H.; Banin, U. "Efficient near-infrared polymer nanocrystals lightemitting diodes", Science 295, 1506-1508, 2002.

(4) Chen, Y. F.; Rosenzweig, Z., "Luminescent CdS quantum dots as selective ion probes", Analytical Chemistry, 74, 5132-5138, 2002.

(5) Joseph, Y.; Besnard, I.; Rosenberger, M.; Guse, B.; Nothofer, H. G.; Wessels, J. M.; Wild, U.; Knop-Gericke, A.; Su, D. S.; Schlogl, R.; Yasuda, A.; Vossmeyer, T., "Self-assembled gold nanoparticle/alkanedithiol films: preparation, electron microscopy, XPS-analysis, charge transport, and vapor-sensing properties", Journal of Physical Chemistry B, 107, 7406-7413, 2003.

(6) Willner, I.; Willner, B., "Functional nanoparticle architectures for sensoric, optoelectronic, and bioelectronic applications" Pure and Applied Chemistry, 74, 1773-1783, 2002.

(7) Huynh, W. U.; Dittmer, J. J.; Alivisatos, A. P. , "Hybrid nanorod-polymer solar cells", Science 295, 2425-2427, 2002.

(8) Sun, B. Q.; Marx, E.; Greenham, N. C., "Photovoltaic devices using blends of branched CdSe nanoparticles and conjugated polymers", Nano Letters, 3, 961-963, 2003.

(9) Klein, D. L.; Roth, R.; Lim, A. K. L.; Alivisatos, A. P.; McEuen, P. L., "A single-electron transistor made from a cadmium selenide nanocrystal", Nature, 389, 699-701, 1997.

(10) Roither.J, Heiss W., Talapin D.V., Gaponik N., Eychmuller A., "Highly directional emission from colloidally synthesized nanocrystals in vertical cavities with small mode spacing", Applied Physics Letters, 84, 2223-2225, 2004 
(11) Finlayson C.E., Ginger D.S., Greenham N.C., "Optical microcavities using luminescent films of semiconductor nanocrystals", Applied Physics Letters, 77, 2500-2502, 2000

(12) Poitras C.B., Lipson M, Du H, Hahn M.A., Krauss T.D., "Photoluminescence enhancement of colloidal quantum dots embedded in a monolithic microcavity", Applied Physics Letters, 82, 4032-4034, 2003

(13) Benisty H., De Neve H., Weisbuch C., "Impact of planar microcavity effects on light extraction", IEEE Journal of Quantum Electronics, 34, 1612-1643, 1998

(14) Klimov V.I., Mikhailovsky A.A., Xu S., Malko A., Hollingsworth J.A., Leatherdale C.A., Eisler H.-J., Bawendi M.G., "Optical gain and stimulated emission in nanocrystals quantum dots", Science, 290, 314-317, 2000

(15) Yoshie T., Scherer A., Hendrickson J., Khitrova G., Gibbs H.M., Rupper G, Ell C., Shchekin O.B., Deppe D.G., "Vacuum Rabi splitting with a single quantum dot in a photonic crystal nanocavity", Nature, 432, 200-203, 2004

(16) Basko D.M., Bassani F., La Rocca G.C., Agranovich V.M., "Electronic energy transfer in a microcavity", Physical Review B, 62, 15962-15977, 2000

(17) Finlayson C.E., Ginger D.S., Greenham N.C., "Enhanced Förster energy transfer in organic/inorganic bilayer optical microcavities", Chemical Physics Letters, 338, 83-87, 2001

(18) Theander M., Granlund T., Johanson D.M., Ruseckas A., Sundström V., Andersson M.R., Inganäs O., "Lasing in a microcavity with an oriented liquid-crystalline polyfluorene copolymer as active layer", Advanced Materials, 13, 323-327, 2001

(19) Martiradonna L., De Vittorio M., Troisi L., Todaro M.T., Mazzeo M., Stomeo T., Anni M., Cingolani R., Gigli G., "Fabrication of hybrid organic-inorganic vertical microcavities through imprint lithography", submitted to Microelectronic Engineering

(20) Heyderman L.J., Schift H., David C., Gobrecht J., Schweitzer T., "Flow behaviour of thin polymer films used for hot embossing lithography", Microelectronic Engineering, 54, 229- 245, 2000

(21) Reiss P., Bleuse J., Pron A., "Highly luminescent CdSe/ZnSe Core/Shell nanocrystals of low size dispersion", Nano letters, 7, 781-784, 2002

(22) Dabbousi B.O., Rodriguez-Viejo J., Mikulec F.V., Heine J.R., Mattoussi H., Ober R., Jensen K.F., Bawendi M.G., "(CdSe) $\mathrm{ZnS}$ core-shell quantum dots: synthesis and characterization of a size series of highly luminescent nanocrystallites", Journal of Physical Chemistry B, 101,9463-9475, 1997

(23) Greenham N.C., Samuel I.D.W., Hayes G.R., Phillips R.T., Kessener Y.A.R.R., Moratti S.C., Holmes A.B., Friend R.H., "Measurement of absolute photoluminescence quantum efficiencies in conjugated polymers", Chemical Physics Letters, 241, 89-96, 1995

(24) Tessler N., Denton G.J., Friend R.H., "Lasing from conjugated-polymer microcavities", Nature, 382, 695-697, 1996

(25) Wenus J., "Tuning the exciton-photon coupling in a strongly coupled organic microcavity containing an optical wedge", Applied Physics Letters, 85, 5848-5850, 2004 